\title{
Metadata Concepts for Advancing the Use of Digital Health Technologies in Clinical Research
}

\author{
Reham Badawy ${ }^{a}$ Farhan Hameed $^{\text {b-d }}$ Lauren Bataille Max A. Little $^{a, f}$ \\ Kasper Claes ${ }^{g}$ Suchi Sariah Jesse M. Cedarbaum ${ }^{\mathrm{i}}$ Diane Stephenson ${ }^{\mathrm{j}}$ \\ Jon Nevillek Walter Maetzler $^{l} \quad$ Alberto J. Espay ${ }^{m}$ Bastiaan R. Bloem ${ }^{n}$ \\ Tanya Simunio Daniel R. Karlin ${ }^{p, q}$
}

\begin{abstract}
aschool of Computer Science, University of Birmingham, Birmingham, UK; ${ }^{b}$ Digital Medicine and Pfizer Innovation Research Lab, Early Clinical Development, Pfizer, Inc., Cambridge, MA, USA; ${ }^{\circ}$ College of Computer and Information Science, Northeastern University, Boston, MA, USA; ${ }^{\mathrm{d}}$ Global Real World Data, Strategy, Analytics \& Informatics (GRWD-SAI), Analytics, Informatics \& Business Intelligence, Chief Digital Office, Pfizer, Inc., New York, NY, USA; ${ }^{\mathrm{e}}$ The Michael J. Fox Foundation for Parkinson's Research, New York, NY, USA; ${ }^{f}$ Media Lab, Massachusetts Institute of

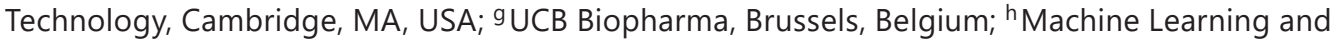
Healthcare Laboratory, Departments of Computer Science, Statistics, and Health Policy, Malone Center for Engineering in Healthcare, and Armstrong Institute for Patient Safety and Quality, Johns Hopkins University, Baltimore, MD, USA; 'Biogen, Cambridge, MA, USA; ${ }^{j}$ Critical Path Institute, Tucson, AZ, USA; ${ }^{\mathrm{C}}$ Clinical Data Interchange Standards Consortium, Austin, TX, USA; 'Department of Neurology, Christian Albrecht University, Kiel, Germany; ${ }^{m}$ James J. and Joan A. Gardner Family Center for Parkinson's Disease and Movement Disorders, University of Cincinnati, Cincinnati, OH, USA; ${ }^{\mathrm{n}}$ Department of Neurology, Donders Institute for Brain, Cognition, and Behavior, Radboud University Medical Center, Nijmegen, The Netherlands; ${ }^{\circ}$ Department of Neurology, Gardner Center for Parkinson's Disease and Movement Disorders, UC Gardner

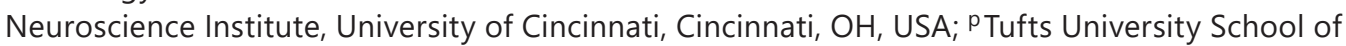
Medicine, Boston, MA, USA; ' HealthMode, New York, NY, USA
\end{abstract}

\section{Keywords}

Digital health technology - Sensors - Objective monitoring of motor symptoms - Metadata . Parkinson's disease

\section{Abstract}

Digital health technologies (smartphones, smartwatches, and other body-worn sensors) can act as novel tools to aid in the diagnosis and remote objective monitoring of an individual's disease symptoms, both in clinical care and in research. Nonetheless, such digital health technologies have yet to widely demonstrate value in clinical research due to insufficient data interpretability and lack of regulatory acceptance. Metadata, i.e., data that accompany and de- 
Badawy et al.: Metadata for Advancing the Use of Digital Health Technologies in Clinical Research

scribe the primary data, can be utilized to better understand the context of the sensor data and can assist in data management, data sharing, and subsequent data analysis. The need for data and metadata standards for digital health technologies has been raised in academic and industry research communities and has also been noted by regulatory authorities. Therefore, to address this unmet need, we here propose a metadata set that reflects regulatory guidelines and that can serve as a conceptual map to (1) inform researchers on the metadata they should collect in digital health studies, aiming to increase the interpretability and exchangeability of their data, and (2) direct standard development organizations on how to extend their existing standards to incorporate digital health technologies. The proposed metadata set is informed by existing standards pertaining to clinical trials and medical devices, in addition to existing schemas that have supported digital health technology studies. We illustrate this specifically in the context of Parkinson's disease, as a model for a wide range of other chronic conditions for which remote monitoring would be useful in both care and science. We invite the scientific and clinical research communities to apply the proposed metadata set to ongoing and planned research. Where the proposed metadata fall short, we ask users to contribute to its ongoing revision so that an adequate degree of consensus can be maintained in a rapidly evolving technology landscape.

(C) 2019 The Author(s)

Published by S. Karger AG, Basel

\section{Introduction}

Digital health technologies such as digital phones and wearables can be used in healthcare and research settings to provide clinicians and clinical trial teams with previously unattainable insights into patients' symptoms, disease progression, and treatment efficacy [1-3] in a cost-effective and efficient manner. We define a digital health technology as containing a sensor or an array of sensors in the environment or to be worn on the person, that enables it to collect objective data on physiological or behavioral phenomena such as heart rate, blood pressure, mobility, speech, sleep patterns, and social engagement [4]. Examples of digital health technologies range from off-the-shelf consumer devices such as smartphones [5-7] and smartwatches [8,9] to medically oriented portable and wearable sensors $[10,11]$. Digital health technologies use a variety of motion and physiological sensors such as accelerometers, gyroscopes, thermometers, and photoplethysmographs. The data collected from these sensors can be processed and analyzed to provide clinically relevant health measurements using computational algorithms $[3,7,12]$. In the clinic, digital health technologies can eliminate much of the subjective bias of clinical measurements. They also allow for remote, long-term monitoring of patient health in naturalistic settings such as the home, and they can do this both continuously and over very long periods of time $[3,7]$. In drug development, data derived from digital health technologies can be employed as surrogate digital outcome measures to potentially increase the statistical power of clinical trials, reduce random sampling issues, and give more detailed insight into response to treatment through more sensitive and frequent measures of disease activity over prolonged periods $[13,14]$.

Parkinson's disease (PD) is a neurodegenerative disorder whose main motor symptoms include bradykinesia, tremor, rigidity, and postural instability [15]. In addition, PD patients can experience a wide range of nonmotor symptoms, including cognitive decline, mood disorders, pain, sleep disturbances, and autonomic dysfunction. The symptoms and signs of PD can fluctuate considerably, both due to variations in the effectiveness of dopaminergic medication (the so-called response fluctuations), but also due to a wide range of other factors (e.g., due to the effects of stress or fatigue). It has been proved very difficult to capture these 
Badawy et al.: Metadata for Advancing the Use of Digital Health Technologies in Clinical Research

fluctuations based on the "snapshot" assessments in clinical practice alone, and the use of conventional diaries is also far from infallible. This disease therefore lends itself well to assessment by digital health technologies $[3,7,16]$. The resulting digital outcome measures or biomarkers may be able to detect effects that are indiscernible in the clinic, increasing the sensitivity to changes of experimental interventions in clinical drug trials. Over the last decade, the PD research community has made progress in investigating the use of digital health technologies as tools for screening clinical and potential premotor (i.e., prodromal) symptoms [10,17], to improve the clinical management of the disease $[18,19]$, and in the assessment of novel therapeutics [20].

Despite the promising application of digital health technologies in research, their usage has been limited in practice due to lack of regulatory guidance [21] on what evidence should be available when validating the precision and reliability of the collected data. These data can be large in size, difficult to manage, and potentially meaningless without appropriate processing and analysis. Clear interpretation of data requires structured data collection and well-defined definitions of the data. Thus, the development of data and metadata standards for digital health technologies can facilitate data management and data pooling to validate their accuracy and interpretability across different research and clinical studies. Nonetheless, little has been done to augment these technologies into existing standards for clinical trials to validate their use in practice. Efforts emphasizing the need to create data standards for digital health technologies have been raised by global regulatory authorities, including the Food and Drug Administration (FDA) [22], the European Medicines Agency Innovation Task Force [23], Health Canada [24], and the Japanese Pharmaceuticals and Medical Devices Agency [25].

More recently, the FDA's Clinical Trials Transformation Initiative developed a comprehensive guide on the use of digital technologies for data capture in clinical trials [26]. Among the many recommendations, the Clinical Trials Transformation Initiative advocates for industry-wide standards related to the collection and reporting of data and emphasizes the need for collecting appropriate metadata to provide sufficient contextual information for interpretability. Metadata, defined here as additional data to accompany and describe the primary data, can be utilized to better understand the context of the collected data $[27,28]$. As such, metadata can help ensure that data from digital health technologies are maximally meaningful and can be reused beyond their primary purpose of collection. Providing metadata specifying information such as the symptom probing task undertaken by a subject, whether monitoring occurred in clinic or at home, and the location of the digital health technology on the subject's body increases the semantic interpretability of the data and the value of algorithms used to extract meaningful information from the data.

In line with existing efforts, we propose a metadata set that reflects regulatory recommendations and that can serve as a conceptual map to (1) inform researchers on the metadata they should be collecting in digital health studies to increase the interpretability and exchangeability of their data, and (2) direct standard development organizations (SDOs) on how their existing standards can be extended to incorporate digital health technologies. The metadata set described here was developed by a diverse working group including data scientists, pharmaceutical companies, and data standard consortium partners, brought together by The Michael J. Fox Foundation for Parkinson's Research (MJFF). While it is designed to provide the PD research community with domain-specific guidance on the generation of digital health data sets suitable for meaningful analyses, it can be used for a wide range of other chronic conditions. 


\section{Literature Review}

\section{Clinical Trials Standards}

Data and metadata standards for clinical research provide a systematic approach to how data are collected and structured. The Clinical Data Interchange Standards Consortium (CDISC) and Health Level 7 (HL7) are two of the most recognized SDOs working in this field. CDISC standards facilitate the traceability and systematic representation of clinical trials at every stage, including the planning phase, data collection, tabulation, statistical analysis, and exchange/sharing of data. The Study Data Tabulation Model (SDTM) [29] is the best-known CDISC standard and is recommended by the FDA for regulatory submissions of clinical trial data. In contrast to CDISC standards, which specifically focus on managing and analyzing clinical trial data, HL7 standards are oriented towards the exchange and retrieval of broader healthcare information captured electronically across various data sources. In particular, the HL7 Fast Healthcare Interoperable Resources (FHIR) framework [30] has been broadly adopted by the healthcare community [31,32] and can aid in monitoring protocol progress, provide greater visibility into trial conduct, and lead to improvements in study efficiency and drug safety. Nonetheless, these standards are not suited to incorporate digital health technologies into clinical trials. Thus, they must be extended appropriately and involve biostatisticians and data scientists in all decisions regarding protocol design, data collection, analysis, and interpretation [26]. This will ensure that the interpretability of data analytical techniques is maximized from the beginning.

\section{Medical Device Standards}

Clinical trials employing the use of medical devices must meet additional regulatory standards to validate and verify the function and safety of the medical devices involved. Medical devices include products such as pacemakers, blood pressure monitors, syringes, MRI scanners, and artificial hips. They may be used to monitor, diagnose, prevent, or treat disease or injury through having a physical or physiological effect on the body. The SDTM Implementation Guide for Medical Devices (SDTMIG-MD) [33] is an extension of the SDTM for structured data collection around device characteristics and events. Another globally recognized and well-adopted standard is the international standard (International Organization for Standardization [ISO]) ISO 14155 [34] for medical device clinical investigations. This standard focuses on good clinical practice for the design, conduct, recording, and reporting of clinical investigations to assess the safety or performance of medical devices for regulatory purposes. Nonetheless, medical devices differ from digital health technologies in their connectivity to information technology, ability to perform remote long-term monitoring outside clinical settings, and their reliance on algorithms for data analysis. Thus, additional metadata, such as the location on which the technology was worn on the body and the time it was taken off, are required to allow for robust analysis of the collected data. Acquiring these metadata is particularly important when deploying digital health technologies in uncontrolled real-world settings with little context.

\section{Sensing Ontologies and Digital Health Schemas}

Focused efforts towards sensors and digital health technologies have been slowly surfacing. The W3C Semantic Sensor Network Ontology (SSNO) and the Sensor, Observation, Sample, and Actuator (SOSA) ontology [35] describe sensors and their observations, the involved procedures, the studied features of interest, and the observed properties. These ontologies are specifically aligned with W3C semantic web technologies to facilitate the major source of sensor data available on the web today, supporting a variety of applications including satellite imagery, social sensing, citizen science, smart cities, and the Web of Things. Nonetheless, these ontologies fail to characterize the specific governance of sensors in healthcare. In parallel to this 
Badawy et al.: Metadata for Advancing the Use of Digital Health Technologies in Clinical Research

work, numerous platforms and smartphone applications in the digital healthcare sector have been propagated on closed-source platforms, creating a patchwork of incompatible applications that serve narrow needs. A major effort towards addressing this challenge is Open mHealth $[36,37]$, an open-architecture software service that exists to support the sustainability and sharing of health data from various and multiple data sources. The Open mHealth API consumes and produces health data that conform to data schemas that specify the format and content of health data, thereby making it easier to process, search, and share data from a variety of data sources. Nonetheless it fails to bridge between existing regulatory standards to accelerate its adoption by regulatory authorities. Moreover, it fails to explain the incentive behind each data schema, thus limiting its adoption and impact in practice.

The overriding aim of the proposed metadata set is to create a conceptual map that bridges the gap between clinical regulatory standards and digital health research to accelerate their use in practice.

\section{Methods}

At the core level, the development of metadata standards requires defining (1) the metadata to collect, (2) the vocabulary to represent the metadata, and (3) how to encode the data for transmission. As an initial step, a working group of 33 experts (see online suppl. material S6; for all online suppl. material, see www.karger.com/doi/10.1159/000502951) was put together by MJFF to define an optimal set of metadata that should accompany primary data collected from digital health technologies for use in PD research. Herein we focus on topic 1, defining the metadata items to serve as a conceptual map for improving the validation and interpretability of digital health technologies for future regulatory approval. We leave topics 2 and 3 for forthcoming discussions based upon future revisions of the proposed metadata set, as evidently these will depend on reaching consensus on topic 1 .

The proposed metadata set, i.e., a curated collection of metadata items used to describe digital health technology-derived data, was derived by expert consensus of the working group, in a structured and standardized process which entailed the following steps:

(1) A day-long face-to-face meeting which brought together all 33 experts of the working group. Through open discussion, it was decided that the metadata would need to describe four important aspects of any study: (a) person, (b) context of collection, (c) observations, and (d) time. The relevant metadata concerning each of these "concepts" were collectively formulated amongst the group in a controlled and iterative process.

(2) Regular virtual meetings to further build on the proposed metadata set. A smaller subset of the working group investigated the structure of existing data and metadata standards including, but not limited to, CDISC, HL7, SSNO, SOSA, ISO 11179, and ISO 8601 [38]. Formal vocabularies used to code data responses in clinical trials such as SNOMED, MeDRA, and LOINC were also investigated. A decision was made to represent the proposed metadata set in the form of a multi-hierarchical tree diagram (mind map) to facilitate the comprehensibility of the representation.

(3) Written feedback on the final proposed metadata set. Members of the working group were invited to provide written feedback on the proposed metadata set that evolved from step 2.

(4) Controlled tracked changes of the following manuscript. The following manuscript underwent several rounds of consensus building by members of the working group to ensure that the metadata set was communicated effectively to the broader PD community.

(5) Formal agreement of the proposed metadata set and manuscript. All authors signed their approval for the final version of the manuscript and the metadata set proposed herein. 
Badawy et al.: Metadata for Advancing the Use of Digital Health Technologies in

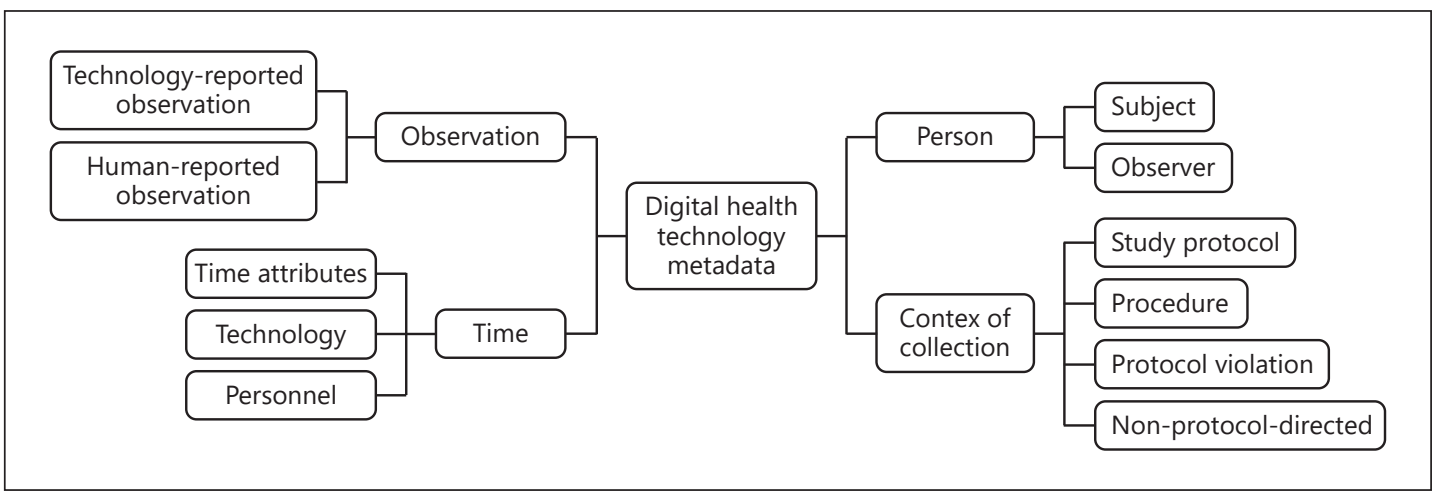

Fig. 1. Overview of the four fundamental concepts of the proposed digital health technology metadata set.

\section{Results}

The metadata set is divided into four main concepts as shown in Figure 1. In developing these concepts, we reviewed existing data and metadata standards for clinical trials, existing sensing ontologies, and the published literature on digital health schemas. This includes, but is not limited to, CDISC, HL7, ISO, W3C, and Open mHealth. The proposed metadata concepts comprise the following: (1) Person: includes relevant metadata pertaining to the individuals who were the subjects or those associated with a subject but not under study. (2) Context of collection: encapsulates relevant metadata relating to a study, its technology, conduct, processes, and procedures. (3) Observation: captures relevant metadata relating to data collected during a study or interpreted through poststudy analyses, including those reported from a digital health technology or a human being. (4) Time: comprises relevant metadata regarding the temporal information about the data collected during the course of primary data collection.

The proposed metadata set adheres to the following properties:

(1) It is a conceptual map that draws from existing standards, without conforming strictly to a specific standard. Thus, it can be easily incorporated within existing internal and international standards. Here, we only list the metadata items that have a technical, data analytical importance and leave it to the user to collect additional metadata that conform to the standard they are following. Where necessary, we introduce new metadata items to fit the proposed application.

(2) Definitions of metadata items are deliberately extensive to facilitate wide application of the proposed metadata set to a variety of use cases utilizing digital health technology for PD. Where appropriate, definitions and encoding of metadata items from existing standards have been adopted and/or modified for our purposes. In instances where metadata items are similar in nature across multiple standards, we favor the use of CDISC terminology and CDISC standards as they are required by global regulatory bodies including the FDA and the Japanese Pharmaceuticals and Medical Devices Agency [39], while the European Medicines Agency strongly recommends their use [40]. Nonetheless, this does not imply universal conformance to CDISC standards, and we subsequently provide sufficient information (including data definitions, motivation, and examples) to allow users and SDOs to map or create the proposed metadata item to other internal or international standards.

(3) We recommend that none of the proposed metadata be regarded as compulsory, as adherence to a "minimum set" of metadata will be dependent on the application of the digital health technology in question and on the available resources of the involved stakeholders. 
Badawy et al.: Metadata for Advancing the Use of Digital Health Technologies in Clinical Research

Table 1. Areas in which existing standards overlap with the observation concept

\begin{tabular}{|c|c|c|c|c|}
\hline $\begin{array}{l}\text { Proposed } \\
\text { concept }\end{array}$ & $\begin{array}{l}\text { Relevant HL7 } \\
\text { FHIR resources }\end{array}$ & $\begin{array}{l}\text { Relevant CDISC SDTMIG-MD } \\
\text { domains }\end{array}$ & $\begin{array}{l}\text { Relevant W3C } \\
\text { SOSA and SSNO } \\
\text { modules }\end{array}$ & $\begin{array}{l}\text { Relevant Open mHealth } \\
\text { schemas }\end{array}$ \\
\hline Observation & $\begin{array}{l}\text { Location } \\
\text { Device } \\
\text { Observation } \\
\text { BodyStructure } \\
\text { DeviceMetric }\end{array}$ & $\begin{array}{l}\text { Study Device Identifiers } \\
\text { Device In-Use } \\
\text { Device Events } \\
\text { Device-Subject Relationships } \\
\text { Device Properties } \\
\text { Device Tracking and Disposition }\end{array}$ & $\begin{array}{l}\text { Observation } \\
\text { Result } \\
\text { Feature } \\
\text { Deployment } \\
\text { System }\end{array}$ & $\begin{array}{l}\text { Data point } \\
\text { Unit value } \\
\text { Body location } \\
\text { Activity name } \\
\text { Position during measurement } \\
\text { Acceleration } \\
\text { Step count }\end{array}$ \\
\hline
\end{tabular}

CDISC, Clinical Data Interchange Standards Consortium; FHIR, Fast Healthcare Interoperable Resources; HL7, Health Level 7; SDTMIG-MD, Study Data Tabulation Model Implementation Guide for Medical Devices; SOSA, Sensor, Observation, Sample, and Actuator; SSNO, Semantic Sensor Network Ontology.

Stakeholders can use the proposed metadata set to agree a priori on the metadata that should be communally collected, thereby ensuring that data are maximally meaningful for analysis when shared.

(4) The proposed metadata set is not disease-specific and may be applied to a variety of neurological disorders utilizing digital health technologies to assess symptoms. While we describe the application of the proposed metadata set in PD, none of the metadata items are specific to the underlying physiology or clinical manifestation of the disease.

Below, we briefly describe each concept. Further details of the proposed metadata set can be found in online supplementary material S1-S5. An interactive, user-friendly version of the complete metadata set along with detailed definitions can be found in online supplementary material S1. A breakdown of each concept with further explanations can be found in online supplementary material S2-S5. We use the term "user" to refer to individuals implementing the metadata set and to distinguish these individuals from those participating in a study, i.e., "subjects."

\section{Observation}

The metadata listed for the observation concept aim to increase the interpretation of the collected data, and as such, reliance on black-box algorithms for data analysis can be minimized. The metadata proposed in this concept are inspired by existing standards including HL7 FHIR, CDISC SDTMIG-MD, W3C SSNO and SOSA, and Open mHealth. Each standard uses different terminology to categorize the collection of information: HL7 groups data into resources, CDISC into domains, W3C SSNO and SOSA into modules, and Open mHealth into data schemas. In Table 1 we list some (but not all) of the existing categories in each standard that have inspired the metadata in the observation concept, thus demonstrating the credibility and harmonization of the proposed metadata items in practice (and with existing standards). While HL7 FHIR and CDISC SDTMIG-MD capture some device-related properties and events, they do not capture the wide range of characteristics unique to digital health data such as sampling frequency. Thus, the observation concept is heavily inspired by W3C SSNO and Open mHealth due to their specialization in capturing sensing data. We add further metadata inspired by the extensive experience of the working group in conducting digital health studies. As the observation concept is our main contribution, we will briefly describe the top-level metadata below. Further lower-level metadata that provide further detail and complement the top-level metadata can be found in online supplementary material S2. 


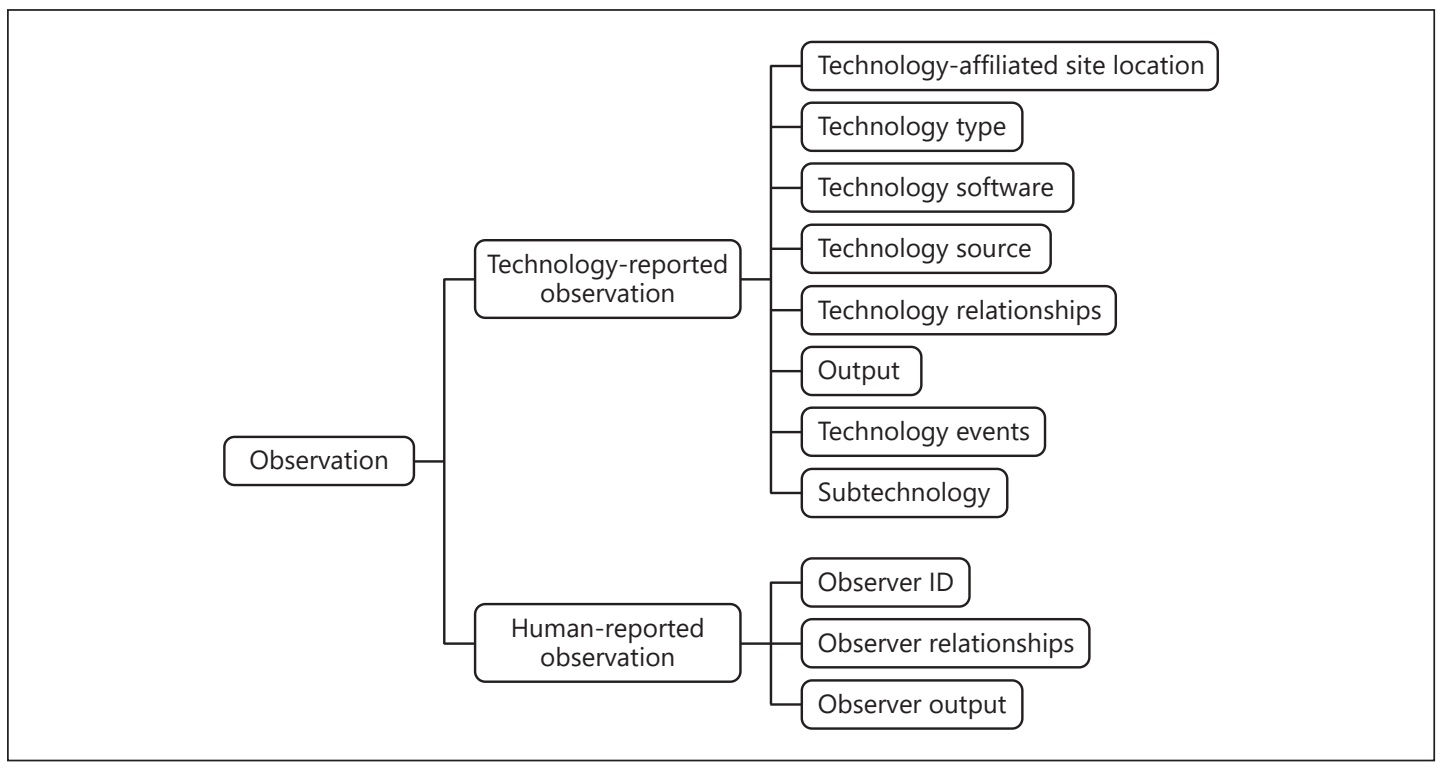

Fig. 2. Overview of the top-level metadata in the observation concept.

The observation concept as shown in Figure 2 includes metadata that describe the data related to other means of measuring or identifying a feature of interest. A feature of interest denotes the thing or event whose property is being measured, estimated, or calculated. For example, a feature of interest could be a subject, the subject's partner, or the temperature of the surrounding environment. Specifically, an observation is the act of carrying out a procedure to measure, estimate, or calculate a value of a property of a feature of interest. For example, it is the recording of a subject's voice while they make a sustained vowel phonation to assess dysphonia, a symptom of PD. The result is then the unprocessed or processed signal from a sensing technology. For example, this could be the sound modulation information collected from a microphone or values collected from an accelerometer.

Observations are split into two high level buckets:

(1) Technology-reported observation relates to characteristics of unprocessed and/or processed data from sensors, such as accelerometer values or processed step counts, respectively. This also includes results collected from subjects documenting or answering questions about symptoms through a software application, e.g., sleep diaries.

(a) Technology-affiliated site location: Describes metadata for linking a technology to a specific geographical site. For example, where there are multiple clinics/institutions involved in a study, each of them will have a unique technology site ID to identify the geographical site/ clinic in which the technology is deployed. This will make it possible for the user to search and extract results from a specific clinic.

(b) Technology type: Describes metadata that define whether a technology is worn on the body as implants/accessories or is a portable/standalone technology. For example, different processing and data analysis techniques may be applied depending on the region on which the digital health technology is worn [41]. This will aid the user in making informed decisions about the nature of the processing applied to the results.

(c) Technology software: Describes metadata relating to the different types of software associated with a technology. For example, a user may only be looking to analyze results from a specific digital operating system version, e.g., iOS 3, as older versions may not be compatible/ adequate for their code/purpose. 
Badawy et al.: Metadata for Advancing the Use of Digital Health Technologies in Clinical Research

(d) Technology source: Describes metadata that outline whether the technology collecting the results is owned (i.e., "bring your own technology") or supplied to the subject. For example, a user may only want to extract results collected from supplied technologies in order to control for technology disparities.

(e) Technology relationships: Describes metadata that define the relationship between a technology and a subject, study, or other technologies. For example, the user may want to search and remove all results from a specific subject because they were found to have an incorrect diagnosis of PD during the course of a study.

(f) Output: Describes metadata about the recorded observations such as sensor precision and units. Denoting the sensor precision, for example, will affect the choice of subsequent data processing.

(g) Technology events: Describes metadata regarding events that may affect the precision and validity of the results, including technology malfunction or maintenance. For example, a user may want to identify and remove intervals in the results in which the technology is charging, undergoing upgrades, or has malfunctioned.

(h) Subtechnologies: Describes metadata that relate to technologies that have been deployed in groups to monitor a feature of interest. In some cases, one defined technology may contain requisite component technologies, which may need to be described separately.

(2) Human-reported observation relates to the results created by individuals associated with a subject. For example, this may be a clinician who is observing the subject to assess their Unified Parkinson's Disease Rating Scale score during the collection of technology-reported observations. It may also be a subject's partner who for example is noting down the subject's behavior while they are asleep. These observations often accompany technology-reported observations to provide comparison (e.g., ground truth) or further clarity (e.g., additional annotations) about the data collected from a technology.

(a) Observer ID: Denotes the unique identifier for the human observer.

(b) Observer relationships: Describes metadata that make clear the relationships associated with an observer, such as the study they are involved in, the procedure that they are asked to carry out, and the subject they are observing.

(c) Observer output: Describes the metadata about the recorded observations made by the observer.

Person

The person concept includes metadata that describe subject characteristics that may play an important factor in disease dynamics, such as age, sex, and medical history. This is particularly important in PD, where there is substantial heterogeneity in the way each patient progresses and responds to medication. Thus, collecting these metadata can improve our understanding of the disease and its manifestation on the subject level during data analysis. Figure 3 shows the top-level metadata proposed for the person concept (for additional complementary lower-level metadata, please see online suppl. material S3). Collecting subject demographics, such as the age and sex of the subject, can allow the user to easily identify observations from subjects within a specific criterion, e.g., males with early-onset PD. Moreover, collecting metadata on concomitant medications may be useful in allowing the user to exclude subjects that are taking a specific medication that might interact with the study intervention. As well as collecting contextual information about the subject, it is also important to do the same for the observer, i.e., those individuals involved in the curation of observations for a subject such as a clinician or the subject's partner. This is useful in assessing the reliability and biases of the collected data.

The metadata proposed for the person concept is inspired mainly by CDISC SDTM and HL7 FHIR, which are well suited for documenting subject information in clinical trials and 
Badawy et al.: Metadata for Advancing the Use of Digital Health Technologies in Clinical Research

Fig. 3. Overview of the top-level

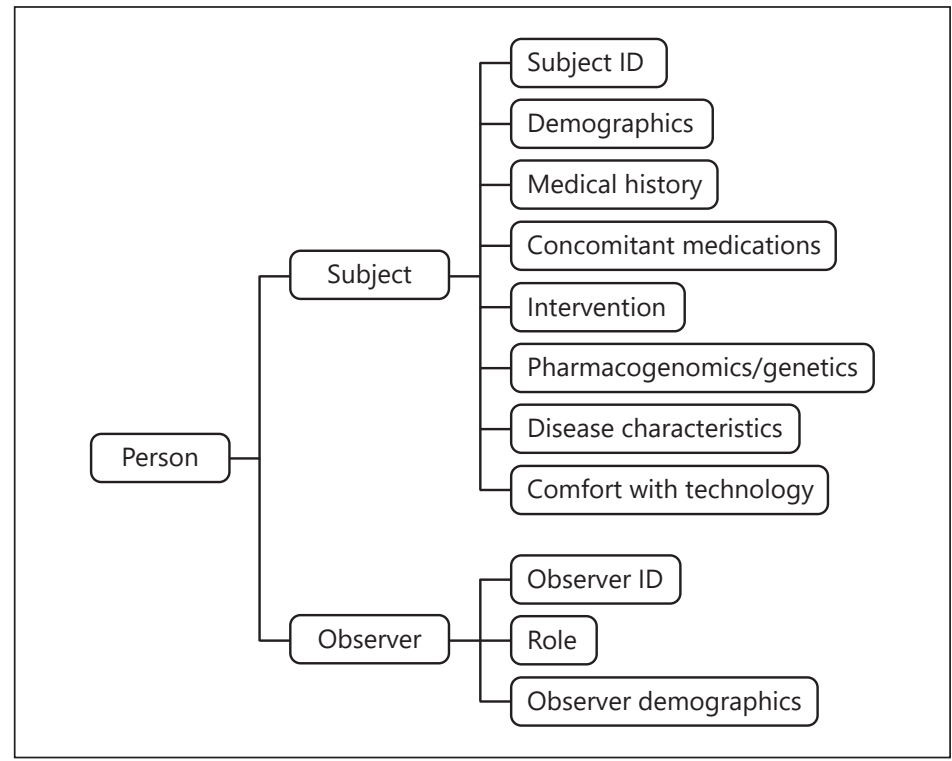
metadata in the person concept.

Table 2. Areas in which existing standards overlap with the person concept

\begin{tabular}{|c|c|c|c|c|}
\hline $\begin{array}{l}\text { Proposed } \\
\text { concept }\end{array}$ & $\begin{array}{l}\text { Relevant HL7 } \\
\text { FHIR resources }\end{array}$ & $\begin{array}{l}\text { Relevant CDISC SDTM/ } \\
\text { SDTMIG-MD domains }\end{array}$ & $\begin{array}{l}\text { Relevant W3C SOSA } \\
\text { and SSNO modules }\end{array}$ & Relevant Open mHealth schemas \\
\hline Person & $\begin{array}{l}\text { Patient } \\
\text { ResearchSubject } \\
\text { Medication } \\
\text { MedicationAdministration }\end{array}$ & $\begin{array}{l}\text { Demographics } \\
\text { Concomitant Medications } \\
\text { Medical History } \\
\text { Associated Person }\end{array}$ & $\mathrm{n} / \mathrm{a}$ & $\begin{array}{l}\text { Body height } \\
\text { Medication adherence } \\
\text { Medication dose unit value } \\
\text { Intervention administration route }\end{array}$ \\
\hline
\end{tabular}

healthcare. These standards cover the collection of a subjects' demographics and medical background as shown in Table 2; nonetheless they lack subject characteristics regarding the use of the technology, such as degree of comfort with technology. We note that while Open mHealth has made extensive efforts to include data schemas that represent information about subjects such as body height, body mass index, body weight, and medication adherence, it does not encompass other important information such as eligibility criteria. Not only is this information important for data contextualization and analysis as discussed in online supplementary material S3, it is also important for regulatory submission. Where necessary, we have developed new metadata items to fit our purposes.

\section{Context of Collection}

In the context of digital health technologies, the study design is of extreme importance as it will govern how the data are to be analyzed. The data cannot be transformed to meaningful information efficiently without knowledge of how they were collected and of the relevant events involved. Thus, the context of collection concept shown in Figure 4; it aims to encapsulate metadata that describe the study, important events that occur during the study, and other relevant contextual information. Collecting metadata on the study protocol provides 
Badawy et al.: Metadata for Advancing the Use of Digital Health Technologies in

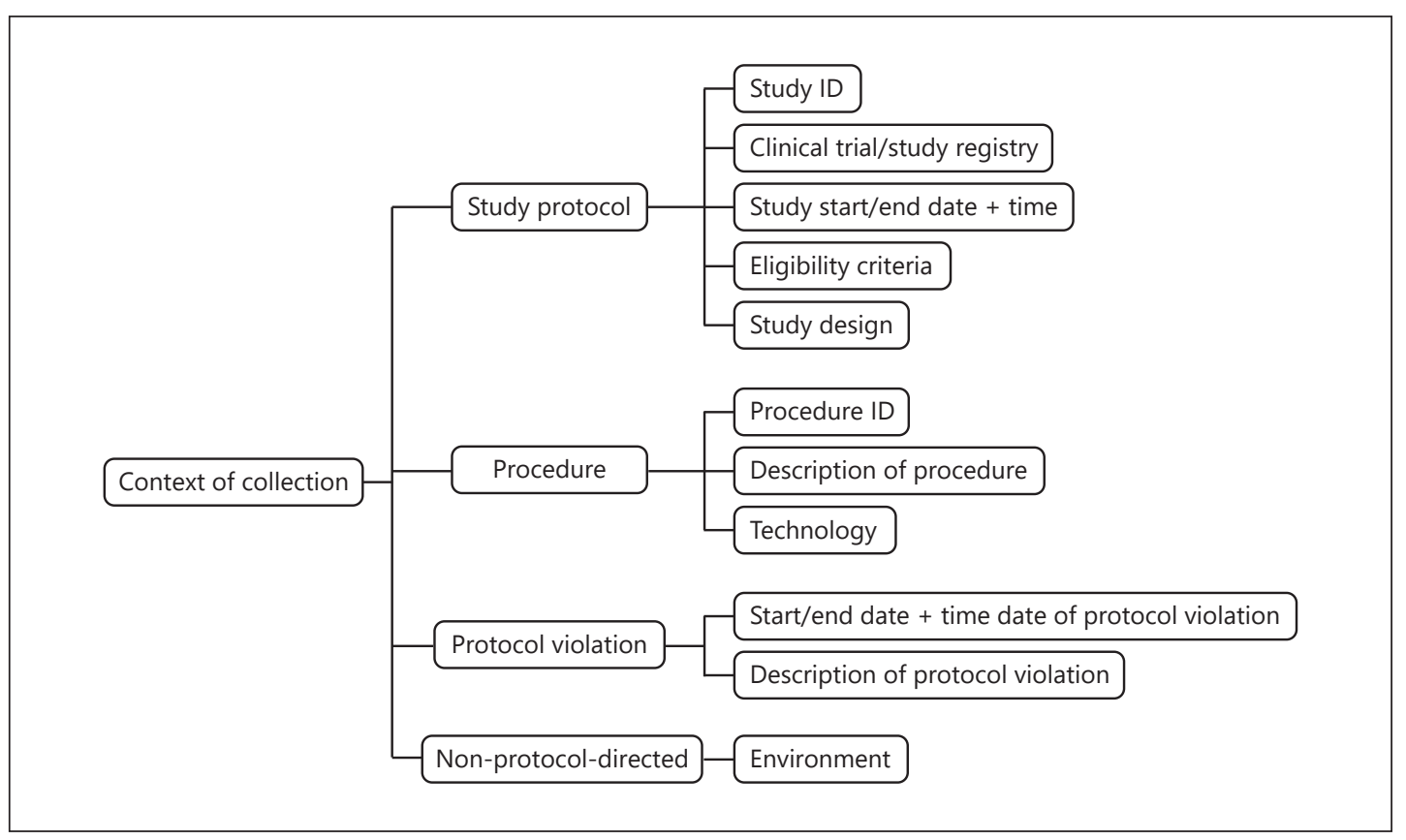

Fig. 4. Overview of the top-level metadata in the context of collection concept.

contextual information about a study. For example, curating metadata on the study design will allow other researchers and clinicians to assess whether the generated data are adequate for their purpose. It is important to point out that the term "study" does not limit the use of the proposed metadata set for research purposes only and may be applied to clinical settings. Moreover, metadata describing the procedure, i.e., a set of instructions that a subject must follow to probe for symptoms, is crucial in making an informed decision about the processing applied to the data. For example, in the case in which a subject is instructed to stand still to investigate postural instability, one would not want to analyze these data for gait information nor would one want to interpret this as involuntary freezing. Furthermore, collecting metadata on protocol violations, i.e., events that significantly depart from the original study protocol, can aid the user in making an informed decision about the quality of the data. It is also important to collect metadata on other non-protocol-directed contextual information that may directly impact the data collected, but which is not covered by the other concepts, such as the environment. The metadata proposed in this concept will ultimately improve data interpretability and allow other researchers and clinicians to assess the quality and reliability of the data for new analyses.

The metadata in this concept are inspired by a range of standards as shown in Table 3, to ensure that the specific clinical, technical, and contextual aspects relating to digital health technologies are accommodated. Due to their heavy use in clinical trials and healthcare, we utilize HL7 FHIR and CDISC SDTM to ensure that structured information about a study/ clinical trial is collected. In addition to this, we also gain inspiration from the CDISC Protocol Representation Model [42], which is a standard for planning and designing clinical research protocols, including study design and eligibility criteria. Again, we provide the motivation behind collecting these metadata from a data pooling and exchangeability perspective in online supplementary material S3 to facilitate their adoption in practice. Furthermore, we utilize CDISC SDTMIG-MD device domains to record information about the digital health technology employed in a study/trial, and add additional metadata of our own to accommodate 
Badawy et al.: Metadata for Advancing the Use of Digital Health Technologies in Clinical Research

Table 3. Areas in which existing standards overlap with the context of collection concept

\begin{tabular}{|c|c|c|c|c|}
\hline $\begin{array}{l}\text { Proposed } \\
\text { concept }\end{array}$ & $\begin{array}{l}\text { Relevant HL7 } \\
\text { FHIR resources }\end{array}$ & $\begin{array}{l}\text { Relevant CDISC PRM/SDTM/ } \\
\text { SDTMIG-MD domains }\end{array}$ & $\begin{array}{l}\text { Relevant W3C SOSA } \\
\text { and SSNO modules }\end{array}$ & $\begin{array}{l}\text { Relevant Open mHealth } \\
\text { schemas }\end{array}$ \\
\hline $\begin{array}{l}\text { Context of } \\
\text { collection }\end{array}$ & $\begin{array}{l}\text { ResearchStudy } \\
\text { ResearchDefinition } \\
\text { Goal } \\
\text { Procedure } \\
\text { Location } \\
\text { Device } \\
\text { Encounter } \\
\text { DetectedIssue } \\
\text { Condition }\end{array}$ & $\begin{array}{l}\text { Trial design (model) } \\
\text { Trial inclusion criteria } \\
\text { Trial summary } \\
\text { Subject visits and elements } \\
\text { Procedures } \\
\text { Protocol deviations } \\
\text { Study device identifiers } \\
\text { Device events }\end{array}$ & Procedure & $\begin{array}{l}\text { Activity name } \\
\text { Ambient temperature } \\
\text { Geoposition } \\
\text { Sleep duration } \\
\text { Sleep episode }\end{array}$ \\
\hline
\end{tabular}

CDISC, Clinical Data Interchange Standards Consortium; FHIR, Fast Healthcare Interoperable Resources; HL7, Health Level 7; PRM, Protocol Representation Model; SDTM, Study Data Tabulation Model; SDTMIG-MD, Study Data Tabulation Model Implementation Guide for Medical Devices; SOSA, Sensor, Observation, Sample, and Actuator; SSNO, Semantic Sensor Network Ontology.

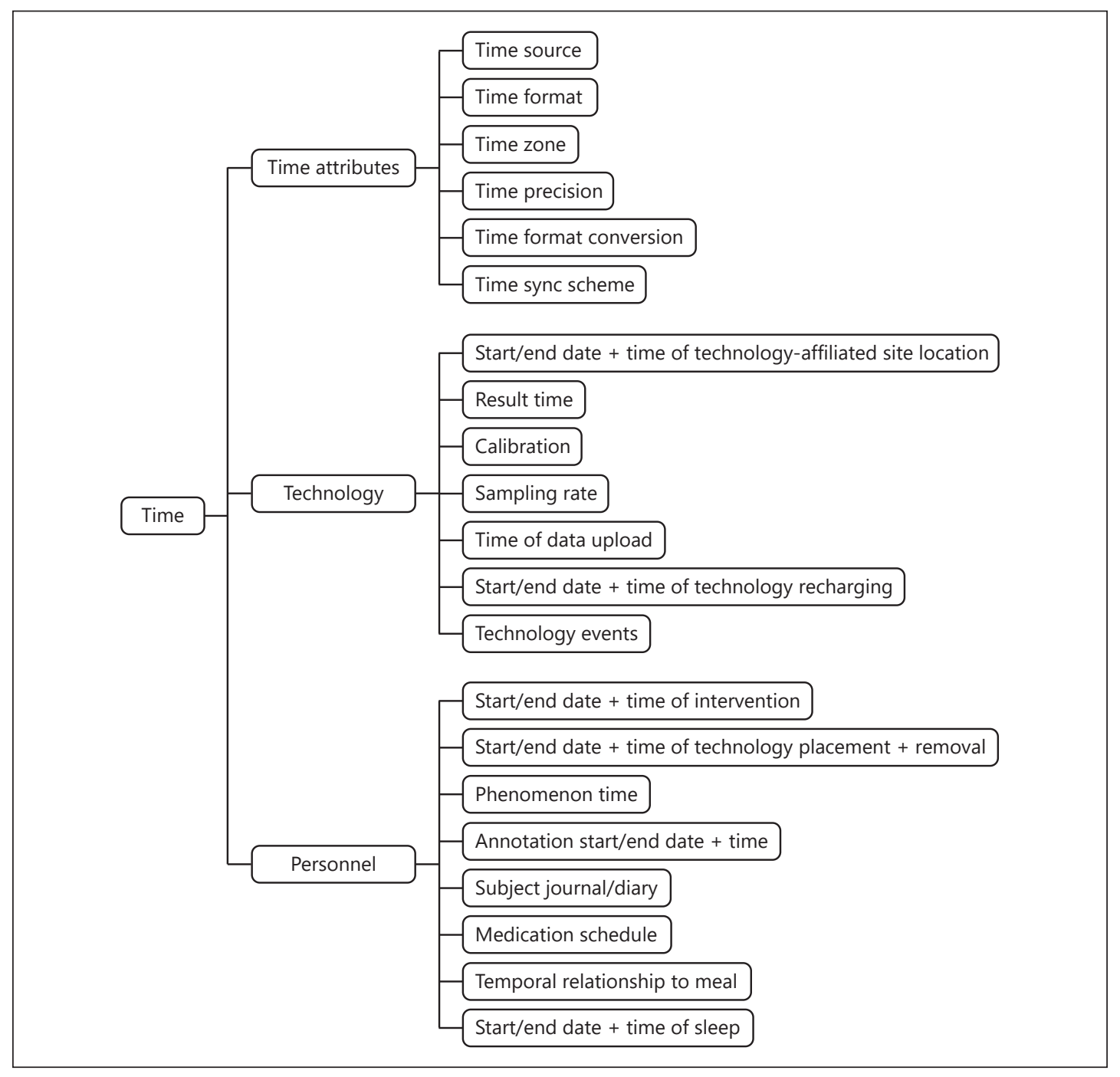

Fig. 5. Overview of the top-level metadata in the time concept. 


\begin{tabular}{l|l}
\hline Digit Biomark 2019;3:116-132 \\
\hline DOI: 10.1159/000502951 & $\begin{array}{l}\text { ○ 2019 The Author(s). Published by S. Karger AG, Basel } \\
\text { www.karger.com/dib }\end{array}$ \\
\hline
\end{tabular}

Badawy et al.: Metadata for Advancing the Use of Digital Health Technologies in Clinical Research

Table 4. Areas in which existing standards overlap with the time concept

\begin{tabular}{lllll}
\hline $\begin{array}{l}\text { Proposed } \\
\text { concept }\end{array}$ & $\begin{array}{l}\text { Relevant HL7 } \\
\text { FHIR resources }\end{array}$ & $\begin{array}{l}\text { Relevant CDISC PRM/SDTM/ } \\
\text { SDTMIG-MD domains }\end{array}$ & $\begin{array}{l}\text { Relevant W3C SOSA } \\
\text { and SSNO modules }\end{array}$ & Relevant Open mHealth schemas \\
\hline Time & $\begin{array}{l}\text { Encompasses } \\
\text { timing variables } \\
\text { within each of the } \\
\text { above resources }\end{array}$ & $\begin{array}{l}\text { Encompasses timing } \\
\text { variables within each of the } \\
\text { above domains }\end{array}$ & $\begin{array}{l}\text { Encompasses timing } \\
\text { variables within } \\
\text { the observation and } \\
\text { result module }\end{array}$ & $\begin{array}{l}\text { Date time } \\
\text { Time interval } \\
\text { Part of day } \\
\text { Patient medication schedule } \\
\text { Temporal relationship to meal } \\
\text { Temporal relationship to physical activity } \\
\end{array}$ \\
& & & Temporal relationship to sleep \\
\hline
\end{tabular}

CDISC, Clinical Data Interchange Standards Consortium; FHIR, Fast Healthcare Interoperable Resources; HL7, Health Level 7; PRM, Protocol Representation Model; SDTM, Study Data Tabulation Model; SDTMIG-MD, Study Data Tabulation Model Implementation Guide for Medical Devices; SOSA, Sensor, Observation, Sample, and Actuator; SSNO, Semantic Sensor Network Ontology.

to their unique features. While clinical and technical metadata are crucial for meaningful interpretation and data analysis, the collection of contextual (non-protocol-directed) metadata that can aid in the exchange of data beyond their original purpose of collection is equally important. We turn to Open mHealth data schemas for inspiration here.

Time

Digital health technologies present the opportunity to more effectively explore disease management and prevention through monitoring health parameters over time and with high temporal resolution. Given that digital health technologies offer broad coverage over time and highly detailed and reliable time information, the concept of time in digital health technology studies is of paramount importance for data processing and analysis. The proposed time concept as shown in Figure 5 aims to emphasize the effect of collecting timestamps for study design-related events and technology attributes that can impact the usability and meaningfulness of the data collected. Understanding the time attributes of a technology, such as the precision and format of the time specifier as collected, is important for assessing data resolution for a particular application and can aid in data processing. Moreover, noting important time events related to the technology, such as the location of a technology during an interval in time, can require certain quality control measures to be applied to the data. For example, data collected outside of the clinic are usually confounded with environmental and behavioral factors that need to be understood and accommodated for during data analysis [43]. Finally, collecting metadata on time-related events that are associated with personnel (i.e., subject), such as time of drug administration, can be useful in assessing the efficacy of a drug by using these time specifiers to identify data from periods immediately prior to and after drug administration. Other time-related events associated with subjects, such as technology placement and removal, can allow users to identify sections of the data that are irrelevant for processing. For further information with examples, see online supplementary material S5.

This concept shares time-related metadata items that preexist in the person, observation, and context of collection concepts. We also include other time-related metadata inspired by Open mHealth data schemas as listed in Table 4, in addition to additional metadata proposed by the working group. We choose to represent all time-related metadata using ISO 8601, which is one of the most widely used standards for representing dates and time. Nonetheless, users are free to choose relevant time standards for their purpose. 
Badawy et al.: Metadata for Advancing the Use of Digital Health Technologies in Clinical Research

\section{Discussion and Conclusion}

The proposed metadata set displays significant overlap with existing data and metadata standards utilized in clinical trials and medical devices to ensure its applicability and regulatory conformity. In addition, it makes use of data schemas and ontologies that have supported digital health technology studies to ensure the robustness and efficacy of the proposed metadata items. Thus, the proposed metadata set highlights how existing standards and schemas can be combined to create a multidisciplinary conceptual map for validating and interpreting digital health technologies for regulatory approval. This will contribute to systematic evaluation of these promising tools in comparable and reproducible clinical studies and subsequently their evaluation and acceptance by healthcare authorities.

We invite the scientific and clinical research communities to engage with the proposed metadata set as an evolving sense of community consensus emerges. The proposed metadata set will be hosted online in the online supplementary material, and members of the scientific and clinical research communities are encouraged to send in their suggestions to the corresponding author. All authors will review these suggestions annually, and updates will be posted online along with a formal document highlighting the changes. In addition to reaching consensus, we list further points of discussion that the scientific community may consider:

(1) Enforced compliance to a minimum set of metadata items. The proposed metadata set does not impose strict adherence to a minimum set of metadata, and we do not discuss the consequences that may arise as a result. While this inherent flexibility may reduce resistance to conformity, we leave it up to the scientific community, particularly to funding bodies and healthcare authorities, to decide whether minimum compliance is a necessary condition in some cases.

(2) Adopting specific vocabulary to represent and encode metadata. We do not describe here how the proposed metadata should be coded, as reaching a consensus on the proposed metadata set is required first and foremost. Moreover, the adopted encoding system will depend on the preexisting encoding system adopted by the SDOs wishing to extend their standards to incorporate digital health technologies. Nonetheless, we advocate for a universal representation of storing metadata, as this will aid in the pooling and exchange of the data among different stakeholders.

(3) Adopting a universal machine-readable format for storage and transmission. Building a machine-readable version of the metadata set will be necessary to identify and retrieve digital data elements from larger data sets to facilitate data management, data sharing, and subsequent analysis. Thus, deciding on a metadata file format will be necessary. We leave it up to the scientific community to decide which of the existing data exchange approaches [30, $44]$ are most appropriate to be used as a universal template.

It is hoped that by involving the PD research community in the iterative development of the proposed metadata set, it will instill ownership and provenance to support its widescale adoption. We hope that discussions spurred by this metadata set will facilitate discussions around metadata representation and encoding to aid SDOs in extending their standards to include digital health technologies for regulatory submission. Ultimately, adoption of the proposed metadata set will depend on its championing by scientists and clinicians designing and executing studies utilizing digital health technologies. 


\section{Acknowledgement}

The authors would like to acknowledge MJFF for bringing together a diverse group of experts to address the lack of metadata standards for digital health technologies in PD. CDISC team colleagues Amy Palmer and Rhonda Facile are recognized for their contributions. The authors would like to extend their gratitude to all 33 members of the working group for their contributions.

\section{Statement of Ethics}

The authors have no ethical conflicts to disclose.

\section{Disclosure Statement}

J.M. Cedarbaum is an employee of and shareholder in Biogen, Inc. W. Maetzler and A.J. Espay are co-chairs of the International Parkinson's and Movement Disorders Society Task Force on Technology. The remaining authors have no conflicts of interest to declare.

\section{Funding Sources}

This work was supported by MJFF (grant ID 14962) to cover the design and writing up of the proposed metadata set and manuscript, respectively.

\section{Author Contributions}

R. Badawy contributed significantly to the curation, design, and modification of the proposed metadata set and developed and wrote the initial manuscript for submission. F. Hameed, D.R. Karlin, and L. Bataille contributed to the conceptualization, design, and modification of the proposed metadata set and were involved in the revision and final approval of the manuscript. M.A. Little, K. Claes, S. Saria, J.M. Cedarbaum, D. Stephenson, J. Neville, W. Maetzler, A.J. Espay, B.R. Bloem, and T. Simuni were involved in critically revising the manuscript for important intellectual content and approved of the final version to be published.

\section{References}

1 Pantelopoulos A, Bourbakis NG. A survey on wearable sensor-based systems for health monitoring and prognosis. IEEE Trans Syst Man Cybern C Appl Rev. 2010 Jan;40(1):1-2.

2 Patel S, Chen BR, Buckley T, Rednic R, McClure D, Tarsy D, et al. Home monitoring of patients with Parkinson's disease via wearable technology and a web-based application. Conf Proc IEEE Eng Med Biol Soc. 2010;2010: 4411-4.

3 Zhan A, Mohan S, Tarolli C, Schneider RB, Adams JL, Sharma S, et al. Using smartphones and machine learning to quantify Parkinson disease severity: the mobile Parkinson disease score. JAMA Neurol. 2018 Jul;75(7): 876-80.

4 Arnerić SP, Cedarbaum JM, Khozin S, Papapetropoulos S, Hill DL, Ropacki M, et al. Biometric monitoring devices for assessing end points in clinical trials: developing an ecosystem. Nat Rev Drug Discov. 2017 Oct; 16(10):736. 


\begin{tabular}{l|l}
\hline \multicolumn{2}{l}{ Digit Biomark 2019;3:116-132 } \\
\hline DOI: 10.1159/000502951 & $\begin{array}{l}\text { (c) 2019 The Author(s). Published by S. Karger AG, Basel } \\
\text { www.karger.com/dib }\end{array}$ \\
\hline
\end{tabular}

Badawy et al.: Metadata for Advancing the Use of Digital Health Technologies in Clinical Research

5 Joundi RA, Brittain JS, Jenkinson N, Green AL, Aziz T. Rapid tremor frequency assessment with the iPhone accelerometer. Parkinsonism Relat Disord. 2011 May;17(4):288-90.

6 Kostikis N, Hristu-Varsakelis D, Arnaoutoglou M, Kotsavasiloglou C. Smartphone-based evaluation of parkinsonian hand tremor: quantitative measurements vs clinical assessment scores. Conf Proc IEEE Eng Med Biol Soc. 2014;2014:906-9.

7 Zhan A, Little MA, Harris DA, Abiola SO, Dorsey ER, Saria S, et al. High frequency remote monitoring of Parkinson's disease via smartphone: platform overview and medication response detection. 2016; 1-12. Available from: https://arxiv.org/abs/1601.00960.

8 Dubey H, Goldberg JC, Abtahi M, Mahler L, Mankodiya K. EchoWear: smartwatch technology for voice and speech treatments of patients with Parkinson's disease. Available from: https://arxiv.org/abs/1612.07608.

9 Wile DJ, Ranawaya R, Kiss ZH. Smart watch accelerometry for analysis and diagnosis of tremor. J Neurosci Methods. 2014 Jun;230:1-4.

10 Andrzejewski KL, Dowling AV, Stamler D, Felong TJ, Harris DA, Wong C, et al. Wearable sensors in Huntington disease: a pilot study. J Huntingtons Dis. 2016 Jun;5(2):199-206.

11 Maetzler W, Domingos J, Srulijes K, Ferreira JJ, Bloem BR. Quantitative wearable sensors for objective assessment of Parkinson's disease. Mov Disord. 2013 Oct;28(12):1628-37.

12 Kubota KJ, Chen JA, Little MA. Machine learning for large-scale wearable sensor data in Parkinson's disease: Concepts, promises, pitfalls, and futures. Mov Disord. 2016 Sep;31(9):1314-26.

13 Izmailova ES, Wagner JA, Perakslis ED. Wearable devices in clinical trials: hype and hypothesis. Clin Pharmacol Ther. 2018 Jul;104(1):42-52.

14 Gossens C. Remote digital biomarker monitoring bringing a smartphone-based diagnostic test for Parkinson's disease progression into an interventional trial. Zenodo; 2015. Available from: https://doi.org/10.5281/ zenodo.31310.

15 Jankovic J. Parkinson's disease: clinical features and diagnosis. J Neurol Neurosurg Psychiatry. 2008 Apr; $79(4): 368-76$.

16 Rovini E, Maremmani C, Cavallo F. How wearable sensors can support Parkinson's disease diagnosis and treatment: a systematic review. Front Neurosci. 2017 Oct; 11:555.

17 Mirelman A, Gurevich T, Giladi N, Bar-Shira A, Orr-Urtreger A, Hausdorff JM. Gait alterations in healthy carriers of the LRRK2 G2019S mutation. Ann Neurol. 2011 Jan;69(1):193-7.

18 Pasluosta CF, Gassner H, Winkler J, Klucken J, Eskofier BM. An emerging era in the management of Parkinson's disease: wearable technologies and the internet of things. IEEE J Biomed Health Inform. 2015 Nov;19(6): 1873-81.

19 Rovini E, Esposito D, Maremmani C, Bongioanni P, Cavallo F. Empowering patients in self-management of Parkinson's disease through cooperative ICT systems. In: Wearable technologies: concepts, methodologies, tools, and applications. IGI Global; 2018. p. 637-63.

20 Henderson EJ, Lord SR, Brodie MA, Gaunt DM, Lawrence AD, Close JC, et al. Rivastigmine for gait stability in patients with Parkinson's disease (ReSPonD): a randomised, double-blind, placebo-controlled, phase 2 trial. Lancet Neurol. 2016 Mar;15(3):249-58.

21 Polhemus AM, Kadhim H, Barnes S, Zebrowski SE, Simmonds A, Masand SN, et al. Accelerating Adoption of Patient-Facing Technologies in Clinical Trials: A Pharmaceutical Industry Perspective on Opportunities and Challenges. Ther Innov Regul Sci. 2019 Jan;53(1):8-24.

22 US Food and Drug Administration. Digital health innovation action plan. Available from: https://www.fda. gov/medical-devices/digital-health [cited June 20, 2019].

23 European Medical Agency. EMA experience with the review of digital technology proposals in medicine development programmes. Available from: https://www.ctti-clinicaltrials.org/sites/www.ctti-clinicaltrials.org/ files/ctti_mobiletechnologies_session6-ema-experience_cerreta_july2018.pdf [cited June 20, 2019].

24 Government of Canada. Notice: Health Canada's Approach to Digital Health Technologies. Available from: https://www.canada.ca/en/health-canada/services/drugs-health-products/medical-devices/activities/ announcements/notice-digital-health-technologies.html [cited June 20, 2019].

25 Lee M, Ly H, Möller CC, Ringel MS. Innovation in Regulatory Science Is Meeting Evolution of Clinical Evidence Generation. Clin Pharmacol Ther. 2019 Apr;105(4):886-98.

26 CTTI. Project: Mobile Technologies. Available from: https://www.ctti-clinicaltrials.org/projects/mobile-technologies [cited June 15, 2019].

27 Duval E. Metadata standards: What, who \& why. J Univers Comput Sci. 2001 Jul;7(7):591-601.

28 Solbrig HR. Metadata and the reintegration of clinical information: ISO 11179. MD Comput. 2000 May-Jun; $17(3): 25-8$.

29 CDISC. Study data tabulation model (SDTM). Available from: https://www.cdisc.org/standards/foundational/sdtm [cited June 5, 2019].

30 HL7. Welcome to FHIR. Available from: https://www.hl7.org/fhir/ [cited June 15, 2019].

31 The Office of the National Coordinator for Health Information Technology. Heat wave: the U.S. is poised to catch FHIR in 2019. Available from: https://www.healthit.gov/buzz-blog/interoperability/heat-wave-the-us-is-poised-to-catch-fhir-in-2019 [cited June 5, 2019].

32 NHS Digital. Fast Healthcare Interoperability Resources: What NHS Digital is doing with FHIR. Available from: https://digital.nhs.uk/services/fhir-apis [cited June 15, 2019]. 
Badawy et al.: Metadata for Advancing the Use of Digital Health Technologies in Clinical Research

33 CDISC. Study Data Tabulation Model Implementation Guide for Medical Devices (SDTMIG-MD). Available from: https://www.cdisc.org/system/files/all/standard_category/application/pdf/stdmig_md_v_1_0. pdf [cited June 15, 2018].

34 ISO. ISO 14155:2011 Clinical investigation of medical devices for human subjects. Available from: https:// www.iso.org/standard/45557.html [cited June 15, 2019].

35 W3C. Semantic Sensor Network Ontology. Available from: https://www.w3.org/TR/vocab-ssn/ [cited June 21, 2019].

36 Open mHealth. The First And Only Open Standard For Mobile Health Data. Available from: http://www.openmhealth.org [cited June 3, 2019].

37 Estrin D, Sim I. Health care delivery. Open mHealth architecture: an engine for health care innovation. Science. 2010 Nov;330(6005):759-60.

38 International Organization for Standardization. Date and time format - ISO 8601 Available from: https:// www.iso.org/iso-8601-date-and-time-format.html [cited June 5, 2019].

39 CDISC. Global Regulatory Requirements. Available from: https://www.cdisc.org/resources/global-regulatory-requirements [cited June 21, 2019].

40 EMA. Final advice to the European Medicines Agency from the clinical trial advisory group on Clinical trial data formats. Available from: https://www.ema.europa.eu/en/documents/other/ctag2-advice-european-medicines-agency-clinical-trial-advisory-group-clinical-trial-data-formats_en-4.pdf [cited June 21, 2019].

41 Atallah L, Lo B, King R, Yang GZ. Sensor placement for activity detection using wearable accelerometers. In: 2010 International Conference on Body Sensor Networks 2010. p. 24-9. Available from: https://doi. org/10.1109/BSN.2010.23.

42 CDISC. Protocol Representation Model. Available from: https://www.cdisc.org/standards/foundational/ protocol [cited June 1, 2019].

43 Badawy R, Raykov YP, Evers LJ, Bloem BR, Faber MJ, Zhan A, et al. Automated quality control for sensor based symptom measurement performed outside the lab. Sensors (Basel). 2018 Apr;18(4):1215.

44 CDISC. ODM-XML. Available from: https://www.cdisc.org/standards/data-exchange/odm [cited June 2, 2019]. 